\title{
Orthodontic retention regimes: will we ever have the answer?
}

\author{
How effective are different retention procedures in maintaining tooth position \\ after treatment by orthodontic appliances?
}

\author{
Littlewood SJ, Millett DT, Doubleday B, Bearn DR, Worthington \\ HV. Retention procedures for stabilising tooth position after treat- \\ ment with orthodontic braces. Cochrane Database of Systematic \\ Reviews 2006; issue 1
}

Data sources The Cochrane Oral Health Group Trials Register, CENTRAL, Medline and Embase were searched to May 2005. Key journals were searched by hand, together with bibliographies of all retrieved papers and review articles. No language restrictions were applied. Authors of trials were contacted to identify unpublished trials.

Study selection Inclusion criteria specified randomised and quasi-randomised controlled trials which involved children or adults who had retainers fitted or adjunctive procedures undertaken, following orthodontic treatment with braces to prevent relapse. The outcomes were amount of relapse, survival of retainers, adverse effects on oral health and quality of life.

Data extraction and synthesis Screening of eligible studies, assessment of the methodological quality of the trials and data extraction were conducted independently and in duplicate. For dichotomous outcomes, the estimate of effect of an intervention was expressed as risk ratios together with $95 \%$ confidence intervals. For continuous outcomes, mean differences and $95 \%$ confidence intervals were used to summarise the data for each group.

Results The search identified 27 articles, of which five met the inclusion criteria. These trials all compared different interventions. There was weak unreliable evidence, based on data from one trial, that a statistically significant increase in stability occurs in both the mandibular $(P<0.001)$ and maxillary anterior segments $(P<0.001)$ when the circumferential supracrestal fiberotomy is used in conjunction with a Hawley retainer, compared with a Hawley retainer alone. There was also weak, unreliable evidence that teeth settle quicker with a Hawley retainer than with a clear overlay retainer after 3 months. The quality of the trial reports was generally poor. No data were available from any study on quality of life assessment. Meta-analysis could not be undertaken as no two studies compared the same retention techniques.

Conclusions There are insufficient research data on which to base our clinical practice on retention at present. There is an urgent need for high quality randomised controlled trials in this crucial area of orthodontic practice.

\section{Commentary}

The orthodontic retention phase has always been an important part of maintaining a good, stable occlusion after active appliance treatment. How many times have orthodontists stressed to patients that the key to an everlasting smile is to, "keep wearing that retainer for as long as you can for an indefinite time frame"? Why have we placed such a burden on our patients?

The authors of this Cochrane Review correctly point out that the evidence in this area of orthodontic research is greatly lacking, something suspected for years. The authors identified five studies to be included in their review but were unable to make comparisons because of the different methods of reporting data. They concluded that more work is needed in this area and suggest a number of possible avenues for investigation. Retention studies, however, are very difficult to perform in a clinical setting. Merely pointing out the lack of evidence does not give us new direction. There are a number of factors to consider and this list is by no means exhaustive:

Compliance issues. How is the researcher to assess the reliability of what the patients say? All seasoned clinicians know that what the patient reports can be different from what they do.

Results and responsibility. What makes a good retention result and what is the time frame for which an orthodontist should be held responsible for the treatment result?

Quality control on the retainers made. What are the criteria in the fabrication and placement of a good appliance?

Cost issues. What is the most cost-effective method for the delivery of care in different health care settings (ie, social systems versus free market fee paying systems)?

Treatment complexity. Different cases start with different levels of complexity. Can researchers standardise this and would there be subjects for the data sets?

Final occlusal results. What was the final occlusion like? Although all clinicians strive for a perfect, settled occlusion, there are situations and circumstances in which these are unachievable. Is the retention strategy greatly affected by this?

Retention studies are multifaceted and require a great deal of planning and resources to carry out, yet the final outcome is probably measured in millimetres and the benefits are questionable. Although we may never find an answer to all these questions, current thinking seems to be reasonable: "Be careful. Wear your retainers well, often and for as long you can". Common sense seems to prevail.

\author{
Chung How Kau \\ Department of Orthodontics, University of Texas Health Science \\ Center, Houston, Texas, USA
}

Evidence-Based Dentistry (2006) 7, 81-82. doi:10.1038/sj.ebd.6400448
Address for correspondence: Luisa M Fernandez Mauleffinch, Cochrane Oral Health Group, MANDEC, School of Dentistry, University of Manchester, Higher Cambridge Street, Manchester M15 6FH, UK. 